\title{
Quality of the clinical laboratory department in a specialized hospital in Alexandria, Egypt
}

\author{
T.A. Elhoseeny ${ }^{7}$ and E.K. Mohammad ${ }^{2}$
}

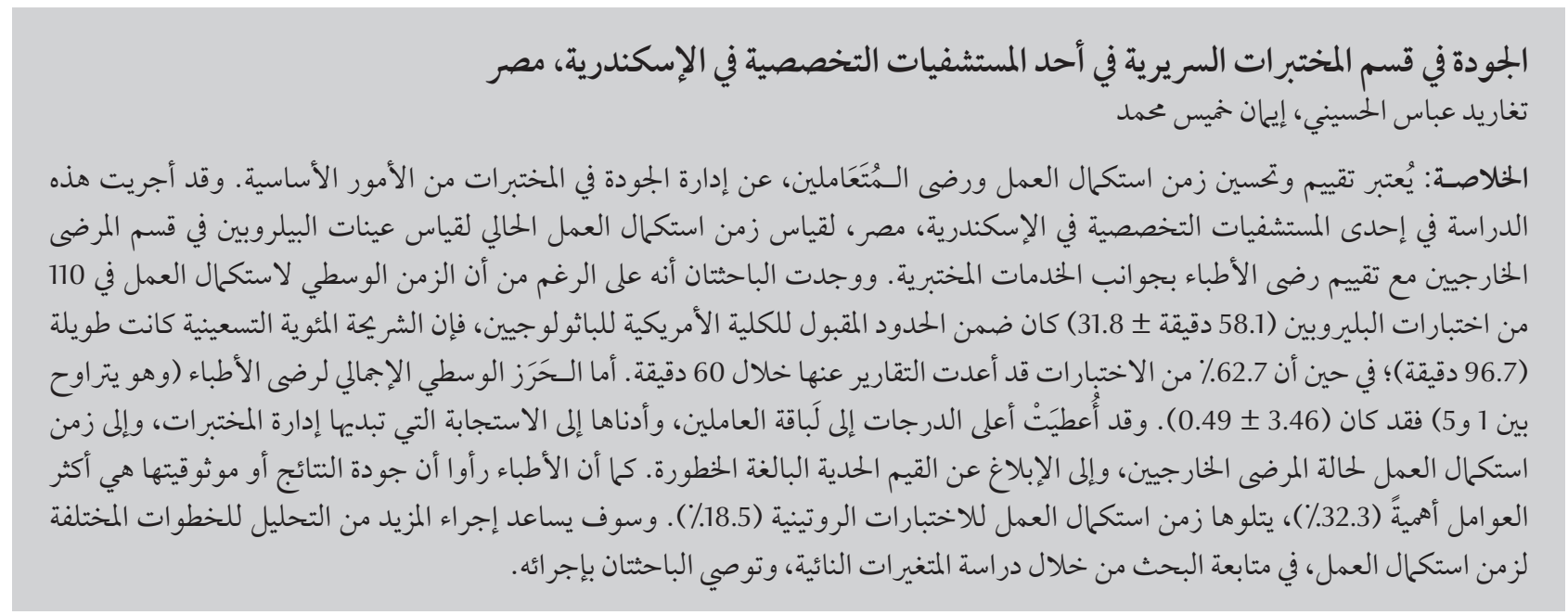

ABSTRACT Assessment and improvement of turnaround times (TAT) as well as customer satisfaction is essential for laboratory quality management. This study in a specialized hospital in Alexandria, Egypt measured the current TAT for outpatient department bilirubin samples and evaluated the satisfaction of physicians with aspects of clinical laboratory services. While the mean TAT for 110 bilirubin tests [58.1 (SD 31.8) min] was within the College of American Pathologists' benchmark, the 90th percentile was long (96.7 min); 62.7\% of tests were reported within $60 \mathrm{~min}$. The mean overall satisfaction score of physicians (range 1-5) was 3.46 (SD 0.49). The highest satisfaction rating was for staff courtesy while the lowest ratings were for laboratory management responsiveness, outpatient stat TAT and critical value notification. Quality or reliability of results was judged by physicians as the most important factor (32.3\%), followed by routine test TAT (18.5\%). Further analysis of the different steps of the TAT would be helpful and follow-up through examining outliers is recommended.

\section{Qualité du service de laboratoire clinique dans un hôpital spécialisé d'Alexandrie (Égypte)}

RÉSUMÉ L'évaluation et l'amélioration des délais de traitement et de la satisfaction du client sont essentielles pour la gestion de la qualité en laboratoire. La présente étude réalisée dans un hôpital spécialisé d'Alexandrie (Égypte) a mesuré le délai de traitement courant des échantillons prélevés en consultation externe et destinés à un dosage de la bilirubine. L'étude a aussi évalué la satisfaction des médecins en termes de services fournis par un laboratoire clinique. Si le délai de traitement moyen de 110 dosages de la bilirubine (58,1 minutes [ET 31,8]) se situait dans la fourchette de référence du College of American Pathologists, le $90^{\text {e }}$ percentile était long (96,7 minutes); 62,7 \% des analyses étaient transmises dans les 60 minutes. Lescore de satisfaction moyen des médecins (extrêmes1-5) était de 3,46 (ET 0,49). Le pourcentage de satisfaction le plus élevé a été décerné à la courtoisie du personnel alors que les résultats les plus faibles ont été attribués à la réactivité de la direction du laboratoire, au délai de traitement des analyses express dans les services de consultations externes et aux notifications ayant une valeur critique. La qualité ou la fiabilité des résultats a été jugée par les médecins comme étant le facteur le plus important (32,3\%), suivi par le délai de traitement des analyses courantes (18,5\%). Des analyses approfondies des différentes étapes du délai de traitement seraient utiles et un suivi des valeurs extrêmes ou aberrantes est recommandé. 


\section{Introduction}

Clinical laboratory testing is an essential element in the delivery of health care services $[1,2]$. The dependence of patient management on laboratory data highlights the need for ensuring the quality of these services $[1,2]$. Delays in reporting laboratory results can cause a concomitant delay in the diagnosis and management of patients [3]. The Joint Commission has underlined this fact by stating that the laboratory is required to systematically assess and improve important functions and work processes and their outcomes [4].

Turnaround time (TAT) is one of the most obvious measures of a laboratory service. Direct assessment of TAT helps managers to understand whether local performance is improving and how it compares with published norms. Despite advances in analytical technology, transport systems and computerization, however, many laboratories have had difficulties improving their TATs [5]. In the USA, TAT has remained at or greater than 1 hour since at least 1965 [6]. Non-analytical delays may be responsible for up to $96 \%$ of total TAT [7]. Investigating causes of outlier TATs that exceeded 70 min showed that only $28 \%$ were caused by the analytic phase of the total testing process; most delays occurred in the pre-analytic steps associated with specimen collection and transport or the post-analytic steps involved with reporting the results [5].

While laboratories have traditionally restricted discussion of quality to technical or analytical quality, focusing on imprecision and inaccuracy goals, clinicians are interested in other dimensions of service quality too. In addition to total test error (imprecision and inaccuracy), this encompasses, availability, cost, relevance and timeliness [8]. Measurement of customer satisfaction brings customer preferences into the quality assessment process and corrects for mistaken assumptions about which particular aspects of services customers value most [9]. Assessing customer satisfaction with laboratory services is required for accreditation by the College of American Pathologists (CAP) and The Joint Commission $[10,11]$. Physicians are one of the primary customers of laboratory services and obtaining their feedback provides laboratory managers with opportunities to identify areas for improvement. The CAP's Q-Probes laboratory quality improvement programme, which has produced numerous publications defining performance benchmarks in pathology and laboratory medicine [12], has provided a standardized survey tool for assessing customer satisfaction with laboratory services [12-14].

Assessment and improvement of TAT as well as assessment of physician satisfaction as a base for improvement efforts is essential for laboratory quality management. The present study in a hospital in Alexandria, Egypt was undertaken to measure the current TAT for outpatient department samples of bilirubin which is a key and common test in the study hospital. The study also attempted to evaluate physician satisfaction overall and with specific aspects of clinical laboratory services.

\section{Methods}

\section{Study setting}

The study was conducted in the laboratory of a 280-bed specialized fever hospital affiliated to the Ministry of Health in Alexandria, Egypt. The hospital has an occupancy rate of $71.5 \%$ [hospital statistics department data]. The hospital laboratory provides clinical chemistry, haematology, coagulation, microbiology, immunology and toxicology services. It provides 200000 tests per year, with approximately $70 \%$ of the results pertaining to inpatients and $30 \%$ to outpatients. The laboratory system consists of a core laboratory that performs the majority of testing for inpatients, with 7 satellites in different buildings of the hospital. The laboratory information system is a paper-only manual system. Blood drawing and sample collection are performed by a phlebotomist in the laboratory department. After analysis of specimens, results are transcribed into a laboratory register and the laboratory reports are transported manually to the requesting physicians.

\section{Sample}

\section{Turnaround times}

The study population for measurement of TAT were outpatient department bilirubin tests requests. The sample size was calculated using Medcalc, version 13.0, based on previous studies on TAT for chemistry specimens [15]. Using a TAT of $68.7 \mathrm{~min}$ and SD 11.3, accepted error of $3 \mathrm{~min}$, an alpha of 0.05 and power $80 \%$, the minimum sample size required was calculated as 112 . Bilirubin test requests from the outpatient department from 09.00-14.00 hours were included until completion of the sample size (14 working days were needed to complete the sample).

\section{Physician satisfaction}

The study population for the assessment of physician satisfaction was all working physicians in the hospital. For measurement of physician satisfaction, all physicians in the hospital $(n=107)$ were included. The response rate was $60.8 \%(n=65)$.

\section{Data collection}

\section{Turnaround times}

For measurement of TAT, it was classified into 3 phases: pre-analytical; analytical; and post-analytical. The preanalytical phase included T1 (waiting time of patient for sampling): starting from patient arrival to the laboratory to the start of processing of test request, $\mathrm{T} 2$ (processing of test request), T3 (collection of blood), T4 (placing of blood in tubes and labelling) and T5 (centrifuge). The analytical phase included $\mathrm{T} 6$ (sample analysis) and T7 (verification of results). The post-analytical phase 
included T8 (transcription of results by physicians) and T9 (reporting of results to physicians). An observational form including these steps was used by one of the researchers to track bilirubin tests and record their TAT.

The laboratory methods used were as follows (Diamond Diagnostics). For total bilirubin: mix the 3 reagents with the specimen and incubate for $10 \mathrm{~min}$ at $20-25^{\circ} \mathrm{C}$ then add the 4 th reagent. Mix and incubate for $5 \mathrm{~min}$ at $20-25^{\circ} \mathrm{C}$; read absorbance of specimen against specimen blank. For direct bilirubin: mix the 2 reagents with $\mathrm{NaCl}(0.9 \%)$ and the specimen and incubate for 5 min at $20-25^{\circ} \mathrm{C}$; read absorbance of specimen against specimen blank. The equipment used for analysis were the BioSystem 310 BTS and the Hetch centrifuge at $4000 \mathrm{rpm}$ for $5 \mathrm{~min}$.

\section{Physician satisfaction}

For measurement of physician satisfaction with the laboratory service a self-administered closed-ended questionnaire designed by CAP [9] was used. The questionnaire was designed to collect data about satisfaction with 15 aspects of clinical laboratory services. Eleven aspects of laboratory services were included. The quality/reliability of test results (which means accuracy and precision of results), accessibility of pathologist, accessibility of laboratory manager, staff courtesy, phlebotomy services, accessibility of laboratory staff, routine test TAT, laboratory management responsiveness, inpatient stat TAT, critical value notification, clinical report format and test menu adequacy. Stat laboratory tests and services are those that are needed immediately in order to manage medical emergencies. Evaluations were provided by physicians using a scale of $1-5(1=$ poor, $2=$ below average, $3=$ average, $4=$ good and 5 = excellent). Physicians were asked to indicate which one of the 11 aspects of service was the most important to them.

\section{Data analysis}

Three composite satisfaction measures were used.

Overall mean satisfaction score $=$ $[($ no of excellent ratings $\times 5)+$ (no. of good ratings $\times 4)+($ no. of average ratings $\times 3)+($ no. of below average ratings $\times 2)+($ no. of poor ratings $\times 1)] /$ total no. of ratings (1-5)

Percentage of excellent or good ratings $=[$ (no. of excellent or good ratings for specific laboratory service category $\times 100)$ ]/total no. of ratings (1-5) for that specific laboratory service category.

Percentage of below average or poor ratings $=[$ no. of below average or poor ratings for specific laboratory service category $\times 100)$ ] /total no. of ratings (1-5) for that specific laboratory service category.

\section{Results}

\section{Turnaround times}

Of 110 laboratory tests $62.7 \%$ tests were reported within $60 \mathrm{~min}, 24.5 \%$ were reported between 60-90 min and the
TAT of $12.7 \%$ (14 tests) was longer than $90 \mathrm{~min}$.

Table 1 shows the mean, median and percentile TAT of the different phases (pre-analytical, analytical and post-analytical) and the total TAT in this outpatient department. The mean TAT was 58.1 (SD 31.8) $\mathrm{min}$. The mean pre-analytical, analytical and post-analytical TATs were 33.6 (SD 25.4), 19.6 (SD 12.3) min and 4.9 (SD 4.2) min respectively. It was found that the 50th percentile (median) of preanalytical, analytical, post-analytical and total TAT were $23 \mathrm{~min}, 16 \mathrm{~min}, 3 \mathrm{~min}$ and 49.5 min respectively while the 90 th percentile of pre-analytical, analytical, post-analytical and total TAT were 67.9 min, $33.9 \mathrm{~min}, 10.9 \mathrm{~min}$ and $96.7 \mathrm{~min}$.

\section{Physician satisfaction}

Table 2 shows the distribution of laboratory service categories according to physicians' ratings. The highest excellent rating of all laboratory service categories was for staff courtesy (43.1\%), which had the highest mean satisfaction score (4.20), followed by quality or reliability of results (21.5\%). The highest poor ratings of the laboratory service categories was for laboratory management responsiveness, outpatient stat TAT and critical value notification (10.8\%, 7.7\%, $7.7 \%)$ respectively. The lowest mean satisfaction score was for outpatient stat TAT and clinical report format (3.26) followed by routine test TAT (3.31).

Table 3 shows that the overall scores of physician satisfaction with

\begin{tabular}{|c|c|c|c|c|c|c|}
\hline \multirow[t]{2}{*}{ Phase } & \multirow[t]{2}{*}{ Mean (SD) } & \multicolumn{5}{|c|}{ Percentiles $^{a}$} \\
\hline & & 10th & 25th & $\begin{array}{c}\text { 50th } \\
\text { (median) }\end{array}$ & 75th & 90th \\
\hline Pre-analytical TAT (min) & $33.6(25.4)$ & 10 & 15 & 23 & 49 & 67.9 \\
\hline Analytical TAT (min) & $19.6(12.3)$ & 7 & 11 & 16 & 26.3 & 33.9 \\
\hline Post-analytical TAT (min) & $4.9(4.2)$ & 2 & 2 & 3 & 7 & 10.9 \\
\hline Total TAT (min) & $58.1(31.8)$ & 24.1 & 35 & 49.5 & 76.3 & 96.7 \\
\hline
\end{tabular}

${ }^{a}$ Higher percentile ranks indicate worse relative performance.

$S D=$ standard deviation. 


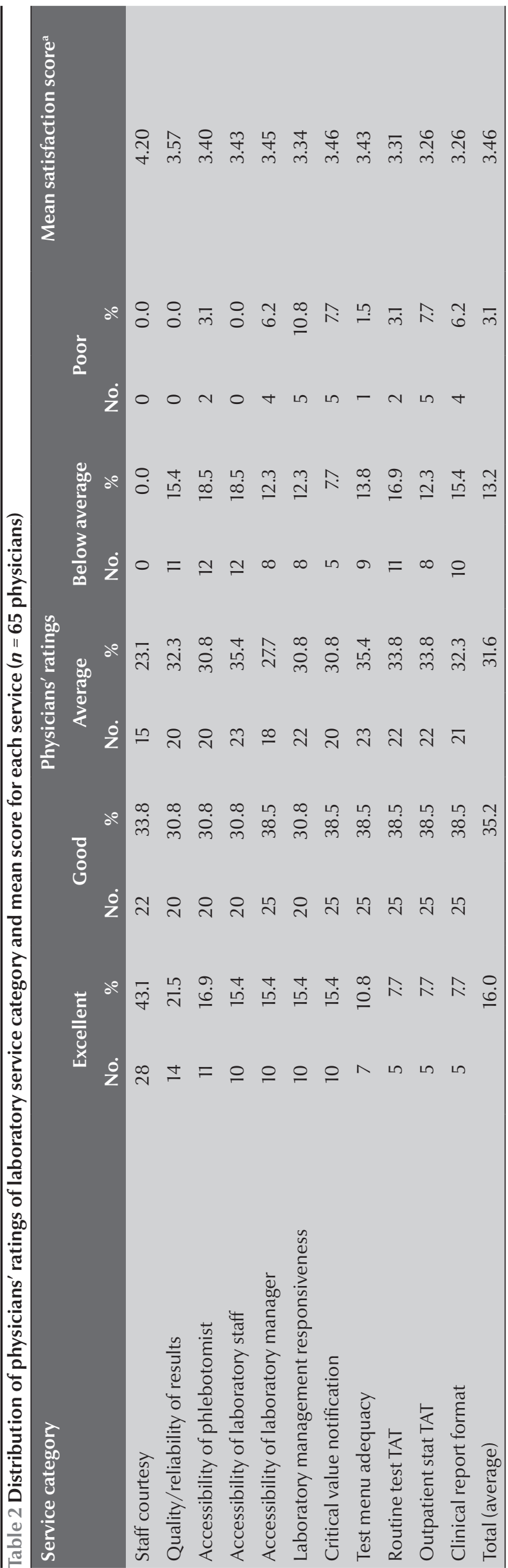

clinical laboratory services ranged from 1.91-4.55 with a mean and median score of 3.5.

Table 4 shows the rankings of physicians' ratings for each laboratory service category. Staff courtesy had the highest percentage of excellent/good ratings (76.9\%). Five service categories received the lowest percentage of excellent/good rating (46.2\%): stat TAT and routine test TAT, laboratory management responsiveness, clinical report format and accessibility of laboratory staff. The laboratory management responsiveness had the highest percentage ratings (23.1\%) for poor/below average. Table 4 also shows the distribution of laboratory service categories according to their importance to the physicians. Quality or reliability of results was considered to be the most important by the physicians (32.3\%) followed by routine test TAT and outpatient stat TAT (18.5\% each) while the least chosen categories were staff courtesy, accessibility of phlebotomist, accessibility of laboratory staff and test menu adequacy ( 1 response, $1.5 \%$ for each).

\section{Discussion}

In the current study we used the mean, the median, the 90th percentile and the outlier rate (percentage of TAT exceeding $60 \mathrm{~min}$ ) to express the TAT. Measuring the mean and the median was important as the TAT was not measured before in the study hospital and we needed to determine our actual time and whether it was within the acceptable measures before starting comparison with external benchmarks and detection of the outliers.

The mean TAT for this fever hospital outpatient department bilirubin testing was 58.1 (SD 31.8) min and the median was $49.5 \mathrm{~min}$. This result seems to be within the acceptable benchmark using TAT goals of $<60 \mathrm{~min}$ for chemical laboratory tests as an initial goal for acceptable TAT according to CAP Q-Probes standards [16]. On the other hand, the 90th percentile of TAT (96.7 min) was much longer than in the 2004 CAP Q-Probes study on biochemical markers of myocardial injury where the 90th percentile of order-to-report TAT for creatine kinase (CK-MB) was $66.5 \mathrm{~min}$ and $61.0 \mathrm{~min}$ for troponin in the emergency department [17]. Also in the current study TAT was longer than that reported by the Asian Medical Center analysis study to measure the TAT of chemistry analytes including bilirubin from the outpatient department, where the 90th percentile of overall TAT (barcode printing to report) was $51.8 \mathrm{~min}$ [15]. 


\begin{tabular}{|c|c|c|c|c|c|c|c|}
\hline \multirow[t]{2}{*}{ Variable } & \multirow[t]{2}{*}{ Mean (SD) } & \multirow[t]{2}{*}{ Range } & \multicolumn{5}{|c|}{ Percentiles } \\
\hline & & & 10th & 25th & $\begin{array}{l}\text { 50th } \\
\text { (median) }\end{array}$ & 75th & 90th \\
\hline $\begin{array}{l}\text { Mean satisfaction } \\
\text { score }^{\mathrm{a}}\end{array}$ & $3.46(0.49)$ & $1.91-4.55$ & 2.84 & 3.18 & 3.45 & 3.86 & 4.04 \\
\hline
\end{tabular}

${ }^{a}$ Range 1-5, where $1=$ poor and $5=$ excellent .

$S D=$ standard deviation

This difference may be attributed to the manual approach of the laboratory in the current study.

The present study defined laboratory TAT as the time taken from patient attendance to laboratory department until the test report became available. The TAT begins with patient arrival at the laboratory then manual registration of his/her data in the laboratory register, preparation for venesection, phlebotomy and labelling of the test-tube. In previous studies these steps were saved through automation so that the patient data were captured through the laboratory information system and using automated barcode printing $[15,17]$. In the Asian Medical Center, laboratory TAT started from barcode printing.

The prolonged 90th percentile is an indicator of high variability among the results. These findings agree with the result that only $62.7 \%$ of specimens (69 test results) were reported within 60 min. This proportion is much less than that reported by the study of the Asian medical centre of South Korea where $98 \%$ of the tests were reported within $60 \min [15]$.

In this study the median pre-analytical phase time was $23 \mathrm{~min}$, the median analytical time was $16 \mathrm{~min}$ and the median post-analytical time was $3 \mathrm{~min}$. It is very useful to analyse the component steps of TAT in it to identify the sources of delay. The pre-analytical phase steps included patient waiting times for sampling, processing of test requests, collection of blood, placing of blood in tubes and labelling and centrifuge. Root cause analysis studies may be needed to identify the sources of delay to enable planning for improvement and reduction of variability, which is a target for quality programmes.
An important point to mention is that the TAT measured here did not include the time taken from the physician order until the patient entered the laboratory. Also reporting of results was considered when the results were documented and the report became ready and not when the result reached the physician. Taking these time periods into consideration could elongate the TAT further.

The mean satisfaction score of physician in this study was 3.46 (SD 0.49) (on a scale of 1-5) and the median was 3.45. This result is not far from 3 studies in the USA, which used the same tool to measure the physician satisfaction and reported a mean satisfaction score between 4.0 and $4.1[13,18,19]$.

The highest excellent rating of laboratory service category was for staff courtesy ( $43.1 \%)$ followed by quality or

\begin{tabular}{|c|c|c|c|}
\hline \multirow[t]{3}{*}{ Service category } & \multicolumn{2}{|c|}{ Physicians' satisfaction ratings } & \multirow{2}{*}{$\begin{array}{l}\text { Most important service } \\
\quad(n=65 \text { physicians })\end{array}$} \\
\hline & $\begin{array}{l}\text { Excellent/ good service } \\
\qquad(n=715 \text { ratings })\end{array}$ & $\begin{array}{l}\text { Poor/ below average service } \\
\text { ( } n=715 \text { ratings })\end{array}$ & \\
\hline & $\%$ & $\%$ & $\%$ \\
\hline Staff courtesy & 76.9 & 0.0 & 1.5 \\
\hline Accessibility of laboratory manager & 53.9 & 18.5 & 6.2 \\
\hline Critical value notification & 53.9 & 15.4 & 10.8 \\
\hline Quality/reliability of results & 52.3 & 15.4 & 32.3 \\
\hline Test menu adequacy & 49.2 & 15.4 & 1.5 \\
\hline Accessibility of phlebotomist & 47.7 & 21.5 & 1.5 \\
\hline Routine test TAT & 46.2 & 20.0 & 18.5 \\
\hline Laboratory management responsiveness & 46.2 & 23.1 & 3.1 \\
\hline Outpatient stat TAT & 46.2 & 20.0 & 18.5 \\
\hline Clinical report format & 46.2 & 21.5 & 4.6 \\
\hline Accessibility of laboratory staff & 46.2 & 18.5 & 1.5 \\
\hline
\end{tabular}

TAT = turnaround time. 
reliability of results (21.5\%), while the highest poor rating was for laboratory management responsiveness, critical value notification and outpatient stat TAT $(10.8 \%, 7.7 \%$ and $7.7 \%$ respectively). Staff courtesy had the highest percentage of excellent/good ratings (76.9\%). Laboratory management responsiveness scored the highest poor/ below average rating (23.1\%). The percentage of poor/below average ratings for routine test TAT and outpatient stat TAT was $20.0 \%$ for each. These findings are comparable with the findings of the Q-Probes programme of the CAP. Staff courtesy had the highest median value (45.2\%) for percentage of excellent ratings among all service categories, followed by quality of results (38.9\%) and critical value notification (38.7\%). Outpatient stat TAT had the highest poor/below average rating (8.8\%), after excluding dimensions that were not included in the current study [13]. The Q-Probes programme in 2002 showed that quality of results had the highest excellent or good rating $(86.7 \%)$, followed by staff courtesy (85.8\%), while outpatient stat TAT also had the highest poor/below average rating (9.5\%) after exclusion of the non-included dimensions in the current study [18]. The surveyed data in 2007 showed that quality/reliability of test results and staff courtesy had the highest median excellent or good rating percentages ( $89.9 \%$ for each), followed by accessibility of laboratory staff $(87.6 \%)$ and accessibility of pathologist (87.5\%). The outpatient stat TAT also had the highest poor/below average rating (5.9\%), after exclusion of the non-included dimensions in the current study [19].

The most important laboratory services for physicians were quality and reliability of results (32.3\%) and routine and outpatient stat TAT (18.5\%). The Q-Probes studies also showed that physicians found quality and reliability of results and TAT the most important aspects of the service $[18,19]$. This high importance of TAT for the physicians can explain our results of low satisfaction with the TAT. The lowest satisfaction scores were given for the routine and stat TATs. We can add the high variability and lack of predictability of the results timing which were demonstrated by the prolonged 90th percentile and the high outlier rate.

\section{Conclusion}

The average TAT of the bilirubin test in our laboratory was within the recommended benchmark of the CAP but the lengthy 90th percentile indicated variability and lack of a standard or expected target for this test. Physicians were satisfied with the courtesy of the laboratory staff but less satisfied with the TAT in general, which is considered to be the second most important aspect of laboratory service.

Further analysis of the different steps of the TAT is needed to identify sources of delay so that planning for improvement can be done. Follow-up measurement of the TAT is recommended by detecting the rate of outliers. Physician satisfaction should be measured frequently to identify opportunities for improvement and as an outcome measure of improvement plans.

\section{References}

1. Shahangian S, Snyder SR. Laboratory medicine quality indicators: a review of the literature. American Journal of Clinical Pathology, 2009, 13:418-431.

2. Chawla R et al. Evaluating laboratory performance with quality indicators. Labmedicine., 2010, 41:297-300.

3. Holland LL, Smith LL, Blick KE. Total laboratory automation can help eliminate the laboratory as a factor in emergency department length of stay. American Journal of Clinical Pathology, 2006, 125:765-770.

4. Comprehensive accreditation manual for pathology and laboratory services. Oakbrook Terrace, Illinois, Joint Commission, 2009.

5. Steindel SJ, Novis DA. Using outlier events to monitor test turnaround time. Archives of Pathology and Laboratory Medicine, 1999, 123:607-614.

6. Howanitz JH, Howanitz PJ. Timeliness as a quality attribute and strategy. American Journal of Clinical Pathology, 2001, 116:311-315

7. Manor PG. Turnaround times in the laboratory: a review of the literature. Clinical Laboratory Science, 1999, 12:85-89.

8. Watts NB. Reproducibility (precision) in alternate site testing. A clinician's perspective. Archives of Pathology and Laboratory Medicine, 1995, 119:914-917.
9. Jones BA et al. Physician satisfaction with clinical laboratory services: College of American Pathologists Q-probes study of 138 institutions. Archives of Pathology and Laboratory Medicine, 2009, 133:38-43.

10. Laboratory general checklist. Laboratory accreditation program. Northfield, Illinois, College of American Pathologists, 2006 (GEN. 20368).

11. Comprehensive accreditation manual for laboratory and point-ofcare testing. Oakbrook Terrace, Illinois, Joint Commission on Accreditation for Healthcare Organizations, 2005-06:PI.1.10.

12. Howanitz PJ. Quality assurance measurements in departments of pathology and laboratory medicine. Archives of Pathology and Laboratory Medicine, 1990, 114:1131-1135.

13. Miller KA, Dale JC. Physician satisfaction with clinical laboratory service, 99-03: Q-probes. Northfield, Illinois, College of American Pathologists, 1999.

14. Jones BA, Walsh MK, Ruby SG. Hospital nursing satisfaction with clinical laboratory services: a College of American Pathologists Q-Probes study of 162 institutions. Archives of Pathology and Laboratory Medicine, 2006, 130:1756-1761.

15. Chung $\mathrm{HJ}$ et al. Analysis of turnaround time by subdividing three phases for outpatient chemistry specimens. Annals of Clinical and Laboratory Science, 2009, 39:144-149. 
16. Lee-Lewandrowski E et al. Utilization and cost analysis of bedside capillary glucose testing in a large teaching hospital: implications for managing point of care testing. American Journal of Medicine, 1994, 97:222-230.

17. Novis DA et al. Biochemical markers of myocardial injury test turnaround time: a College of American Pathologists Q-Probes study of 7020 troponin and 4368 creatine kinase-MB determinations in 159 institutions. Archives of Pathology \& Laboratory Medicine, 2004, 128:158-164.
18. Howanitz PJ. Physician satisfaction with clinical laboratory services: Q-probes data analysis and critique. Northfield, Illinois, College of American Pathologists, 2002.

19. Jones BA, Bekeris LG. Physician satisfaction with clinical laboratory services: College of American Pathologists, Q-probes study of 138 institutions. Archives of Pathology and Laboratory Medicine, 2009, 133:38-43.

\section{Quality assurance in laboratories}

The maintenance of a quality management system is crucial to a laboratory for providing the correct test results every time. Important elements of a quality management system include:

- Documentation

- $\quad$ Standard Operating Procedures (SOPs)

- Quality control samples

- $\quad$ External Quality Assessment Scheme

Quality control procedures are used in each assay to assure a test run is valid and the resultsobtained are reliable; these procedures include the use of kit controls and quality control samples.

External quality assessment schemes aim to analyse the accuracy of the entire testing process from receipt of sample and testing of sample to reporting of results.

Source: www.who.int/diagnostics_laboratory/quality/en/ 